\title{
Características Médias do vento acima e abaixo do dossel da floresta durante o GoAmazon em um sítio experimental na Amazônia
}

\author{
Wind averages features above and below of the forest canopy during GoAmazon \\ in an experimental site in the Amazon
}

Raoni Aquino Silva de Santana ${ }^{1,2}$, Roseilson Souza do Vale1,2, Julio Tóta ${ }^{3}$, Rosa Maria Nascimento dos Santos, David Roy Fitzjarrald5, Giorgio Arlan da Silva Picanço ${ }^{6}$, Sarah Suely Alves. Batalha ${ }^{6}$, Ana

Carla dos Santos Gomes ${ }^{3}$, Gabriel Brito Costa ${ }^{8}$, Raphael Tapajós ${ }^{1}$ e Rodrigo da Silva ${ }^{3}$ raoniass@gmail.com; roseilsondovale@gmail.com; totaju@gmail.com; rosa.maria.sto@gmail.com; dfitzjarrald@albany.edu; giorgiopicanco@gmail.com; ssa.batalha@gmail.com; anacarlasg02@gmail.com; gabrielbritocosta@gmail.com; rpablotapajos@gmail.com; rsilvf@gmail.com

\footnotetext{
${ }^{1}$ Mestre, Instituto de Engenharia e Geociências, Universidade Federal do Oeste do Pará, Santarém, Brasil 2Pós-Graduação em Clima e Ambiente, Instituto Nacional de Pesquisas da Amazônia e Universidade do Estado do Amazonas, Manaus, Brasil

${ }^{3}$ Doutor(a), Instituto de Engenharia e Geociências, Universidade Federal do Oeste do Pará, Santarém, Brasil ${ }^{4}$ Doutora, Escola Superior de Tecnologia, Universidade do Estado do Amazonas, Manaus, Brasil ${ }^{5} \mathrm{PhD}$, University at Albany, USA

${ }^{6}$ Graduando (PIBIT), Instituto de Engenharia e Geociências, Universidade Federal do Oeste do Pará, Santarém, Brasil

${ }^{7}$ Mestre, Programa de Pós-graduação Doutorado em Sociedade, Naturezae Desenvolvimento, Santarém, Brasil

${ }^{8}$ Doutor(a), Instituto de Biodiversidade e Florestas, Universidade Federal do Oeste do Pará, Santarém, Brasil
}

\begin{abstract}
Resumo
O objetivo deste trabalho foi avaliar as características médias da evolução temporal do perfil vertical da velocidade e direção do vento acima e abaixo do dossel da floresta no sítio experimental do Cuieiras, localizado cerca de $100 \mathrm{Km}$ da cidade Manaus, estado do Amazonas, Brasil. Para tanto, foram utilizados aproximadamente 10 meses de dados de 10 anemômetros sônicos instalados em diferentes níveis acima e abaixo do dossel da floresta, durante o projeto GoAmazon, no ano de 2014 . Analisando o conjunto de dados, por meio de médias para cada 30 minutos diários de todo período estudado, foi possível observar que o perfil vertical da velocidade do vento varia de acordo com as diferentes camadas acima e abaixo do dossel da floresta e possui uma forma tipo "S", definida pelas obstruções físicas impostas pela heterogeneidade vertical da floresta. As direções predominantes do vento não são constantes com a altura, sugerindo que circulações locais podem influenciar o escoamento no sítio experimental estudado.
\end{abstract}

Palavras-chave: Perfil, Vento, Floresta, Amazônia, GoAmazon.

\begin{abstract}
The aim of this study was to evaluate the temporal evolution of average characteristics of the vertical profiles of speed and direction above and within of the forest canopy at the Cuieiras experimental site, located about $100 \mathrm{~km}$ from the Manaus, Amazonas state, Brazil. We used about 10 months of half-hourly averaged data from 10 sonic anemometers installed at different levels above and within the forest canopy during GoAmazon project in 2014. We found that the vertical wind speed profile varied with the various layers above and below the forest canopy, describing an S-shape type, a form determined by physical obstructions imposed by vertical heterogeneity of the canopy. The predominant wind directions were not constant with height, suggesting that very local circulations can influence the subcanopy flow.
\end{abstract}

Keywords: Profile, Wind, Forest, Amazon, GoAmazon. 


\section{Introdução}

O estudo das trocas de energia, massa e momentum entre a superfície terrestre e a atmosfera é parte essencial no entendimento dos processos que ocorrem na interação biosferaatmosfera. Em particular, as regiões de florestas tropicais, especialmente a floresta amazônica, exercem papel fundamental nessas trocas, dada a sua importância no balanço global de carbono (Melillo et al., 1993; Malhi et al., 1998) e transferência de umidade do solo e por interceptação para atmosfera (Shuttleworth, 1988), por exemplo.

O transporte de energia e quantidades escalares $\left(\mathrm{CO}_{2}\right.$, por exemplo) da floresta para camadas superiores da atmosfera é realizada por movimentos verticais turbulentos. Tais movimentos, por sua vez, podem ser gerados térmica ou mecanicamente. Em regiões de floresta densa, como a floresta Amazônica, pouca radiação solar incidente consegue atingir o solo (cerca de 1 a $3 \%$, segundo Shuttleworth et al., 1984), o resultado disso é que abaixo do dossel da floresta forma-se uma camada atmosférica estaticamente estável em média, em outas palavras, o ambiente abaixo do dossel é desacoplado da parte superior da floresta (Fitzjarrald et al., 1990). Este desacoplamento pode gerar camadas de ar fisicamente distintas.

De acordo com Yi et al. (2005) e Yi (2008) uma das variáveis fundamentais para se entender os processos de trocas citadas acima é o perfil vertical da velocidade do vento, que segundo os autores pode apresentar um formato de "S" ("S-shape"). Baseado nisso, este trabalho propõe estudar as características médias do perfil vertical do vento em um sítio experimental de floresta densa na Amazônia.

\section{Material e Métodos}

Os dados utilizados nesta pesquisa foram coletados no sítio experimental K34, situado na reserva biológica do Cuieiras ( $\left.54^{\circ} 58^{\prime} \mathrm{O}, 2^{\circ} 51^{\prime} \mathrm{S}\right)$, distante cerca de $100 \mathrm{Km}$ a noroeste da cidade de Manaus, a qual pertencente ao estado do Amazonas, Brasil. Neste local está instalada uma torre de medidas micrometeorológicas, com área de $1,5 \mathrm{~m} \mathrm{X} 2,5 \mathrm{~m}$ de seção transversal e $50 \mathrm{~m}$ de altura, tal torre foi implementada no ano de 1999 e desde então vem sendo utilizada como ponto de observação de longo prazo na Amazônia (mais detalhes sobre estes dados é encontrado em Araújo et al. (2002)).

As medidas ocorreram durante os meses de março a dezembro de 2014, como parte do projeto GoAmazon (do inglês, "Green Ocean Amazon", Oceano Verde Amazônico), consistindo, basicamente, de perfis verticais das componentes de velocidade do vento (vertical, zonal e meridional) e temperatura coletados por anemômetros sônicos tridimensionais (CSAT3, Campbell Scientific). Os anemômetros sônicos foram instalados em 10 níveis diferentes, dentro e acima do dossel da floresta, sendo 9 destes instalados na torre principal de $50 \mathrm{~m}$, nas alturas de $6,13,19,25,28,32,35$, 39 e $43 \mathrm{~m}$, além disso, o anemômetro sônico mais próximo ao solo $(1,5 \mathrm{~m}$ de altura) foi instalado em uma pequena torre distante cerca de 30 metros da torre principal (Figura 1).

A partir dos dados das componentes do vento obteve-se a velocidade horizontal e a direção do vento, depois de removidos picos irreais, tomando como base o diagnóstico fornecido pelo instrumento de medida. Em seguida, foi realizada a média para cada 30 minutos diários para todo o conjunto de dados utilizados nesta pesquisa. 


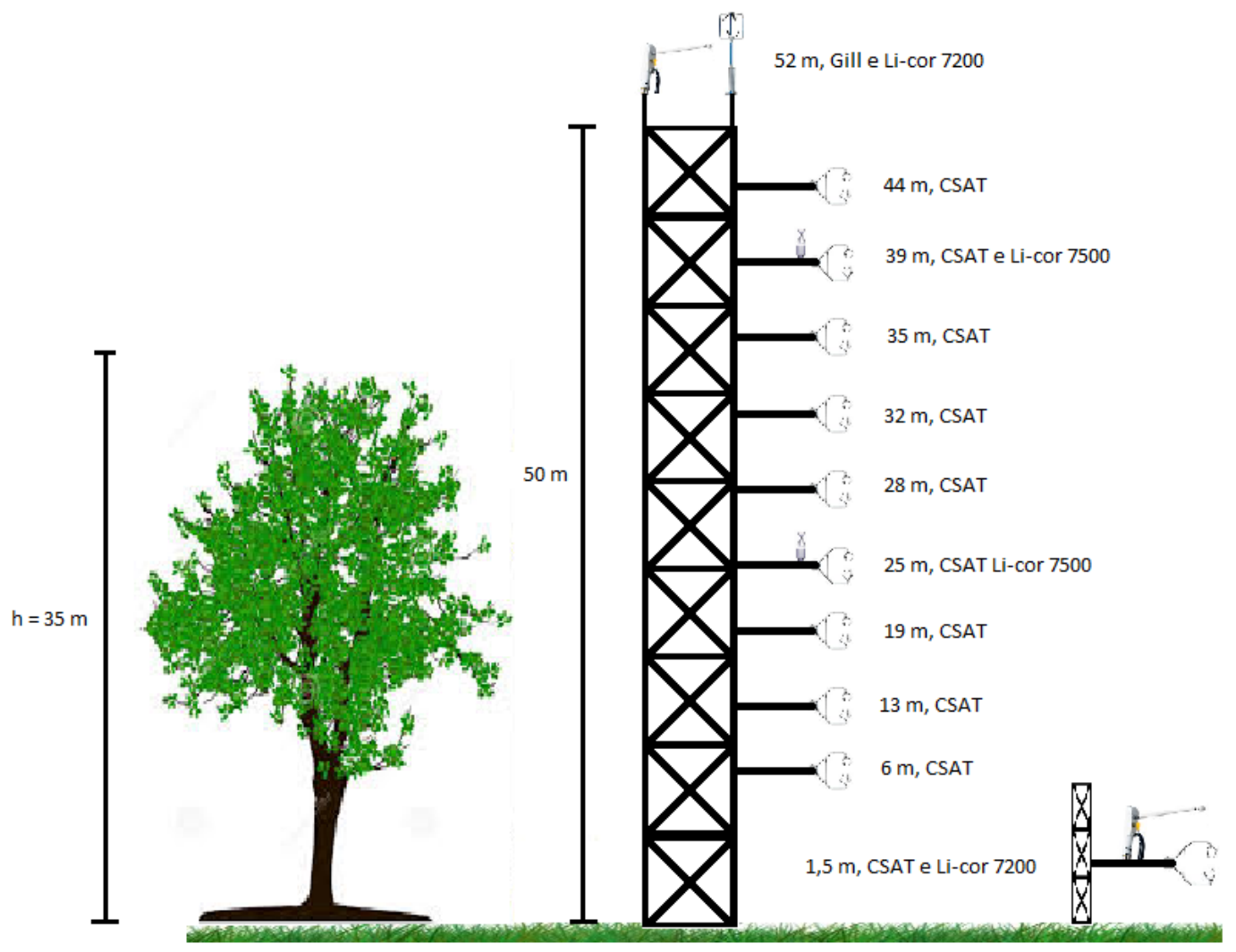

Figura 1. Figura ilustrativa representando os instrumentos de medidas instalados na K34 durante os meses de março a dezembro de 2014.

\section{Resultados e discussão}

Dividindo-se o perfil vertical da floresta em três regiões: sub-dossel; camada do dossel e atmosfera acima do dossel, notou-se uma nítida diferença no escoamento entre estas camadas (Figura 2). A velocidade do vento foi em média bastante fraca, os maiores valores foram observados na altura de 1,2 da altura do dossel (sendo $\mathrm{h}=35 \mathrm{~m}$ a altura média da copa), entre os horários de 10 a 15 horas (hora local, HL), atingindo o valor aproximado de $2 \mathrm{~ms}^{-1}$. Na camada do dossel a velocidade do vento se aproxima de zero durante a noite e oscila em torno de $0,5 \mathrm{~ms}^{-1}$ durante o dia. Já na camada do sub-dossel, a velocidade do vento volta se intensificar, porém com valores baixos, quando comparados com os observados na camada acima do dossel. A configuração do perfil vertical da velocidade do vento no sítio da K34 assemelha-se ao perfil com formato de " $\mathrm{S}$ " sugerido por Yi (2008), sendo a obstrução física imposta pela floresta, provavelmente, o que define este tipo de perfil.

Em relação à evolução temporal do perfil vertical de direção do vento (Figura 2, painel inferior), foi possível observar que nem todas as camadas de ar escoam nas mesmas direções. Acima do dossel da floresta, a direção predominante foi de sudeste, tanto durante o dia quanto à noite, um resultado similar ao encontrado por Tóta et al. (2012) para o ano de 2006, no mesmo sítio experimental estudado. Em torno de 0,4 de $h$ a direção predominante foi de sul a sudoeste em praticamente todos os horários diários. A exceção ocorreu por volta das $7 \mathrm{HL}$, em que o perfil vertical de direção do vento se aproxima da homogeneidade em todas as alturas. O mesmo comportamento também foi observado abaixo de 0,2 de $h$ entre os horários de 11 a 21 HL. Uma primeira explicação para o comportamento da direção do vento nas diferentes camadas de ar é a 
possibilidade de os sensores estarem levemente desalinhados, devido à dificuldade de instalação destes na floresta densa. A segunda, é que circulações locais podem ocorrer devido à heterogeneidade do terreno do sítio K34 (Tóta et al., 2012).
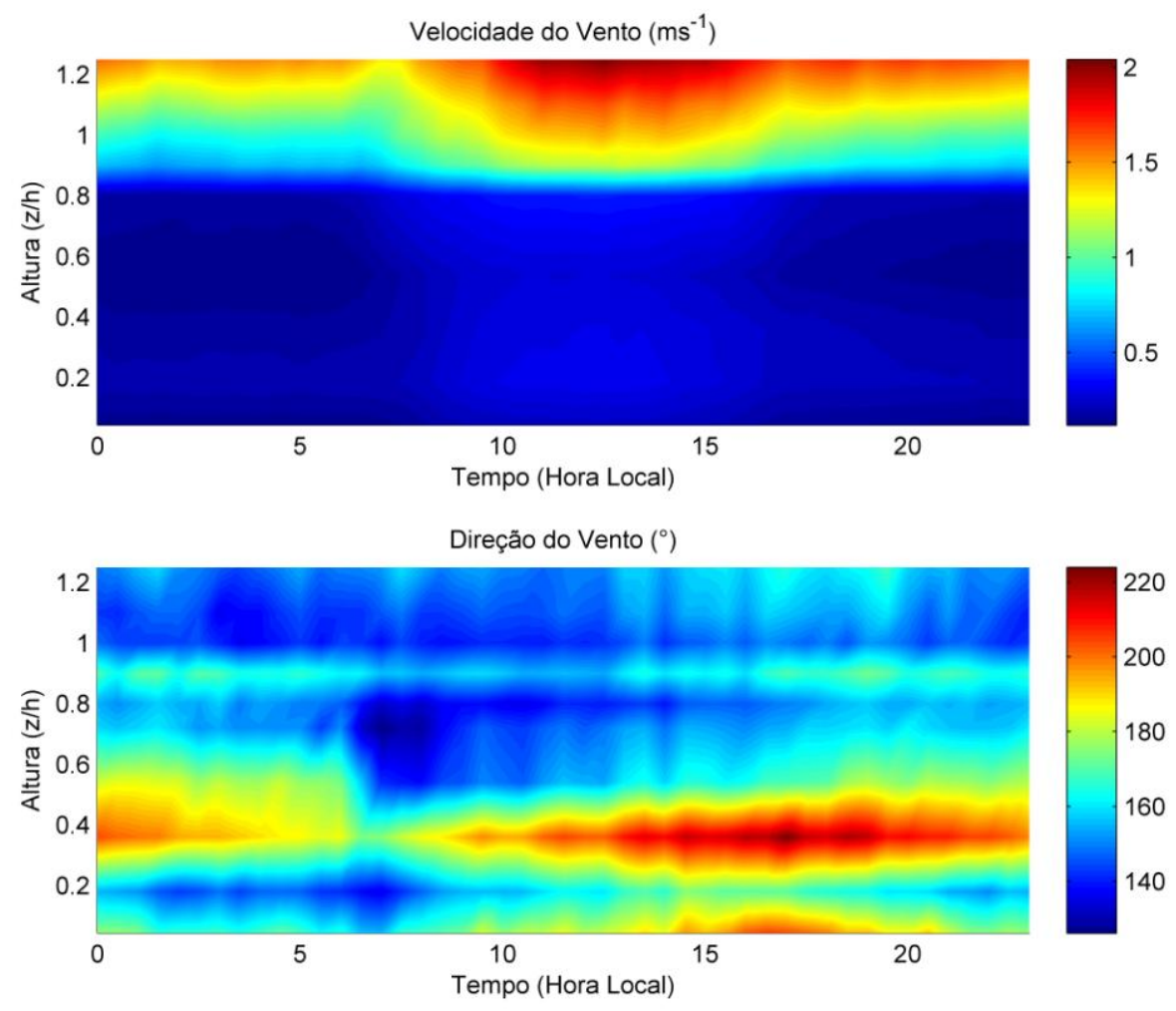

Figura 2. Padrão médio diário do perfil vertical da velocidade (painel superior) e direção (painel inferior) do vento. No eixo y as alturas das medições $(\mathrm{z})$ foram normalizadas com a altura média do dossel $(\mathrm{h}=35$ $\mathrm{m})$.

\section{Conclusões}

Neste trabalho foram utilizados cerca de 10 meses de dados de 10 anemômetros sônicos instalados em diversas alturas acima e abaixo do dossel da floresta durante o ano de 2014. foi possível observar que o perfil vertical da velocidade do vento varia de acordo com as diferentes camadas acima e abaixo do dossel da floresta e possui uma forma tipo " $S$ ", definida pelas obstruções físicas impostas pela heterogeneidade vertical da floresta. As direções predominantes do vento não são constantes com a altura, sugerindo que circulações locais podem influenciar o escoamento no sítio experimental estudado.

\section{Agradecimentos}

Como primeiro autor, agradeço a CAPES pela bolsa de doutorado concedida. Os autores agradecem também a todos aqueles que participaram do projeto GoAmazon no sítio da K34, desde os coordenadores até aos motoristas e caseiros que possibilitaram a coleta dos dados utilizados neste trabalho. 


\section{Referências}

Araújo, A. C.; Nobre, A. D.; Kruijt, B.; Elbers, J. A.; Dallarosa, R.; Stefani, P.; von Randow, C.; Manzi, A. O.; Culf, A. D.; J. Gash, H. C.; Valentini, R.; Kabat, P. Comparative measurements of carbon dioxide fluxes from two nearby towers in a central Amazonian rainforest: The Manaus LBA site, Journal of Geophysical Research. v. 107, n. D20, p. 80909010, 2002.

Fitzjarrald, D. R.; Moore, K. E. Mechanisms of nocturnal exchange between the rain-forest and the atmosphere, Journal of Geophysical Research. v. 95, n. D10, p. 16.839-16.850, 1990.

Malhi, Y.; Nobre, A. D.; Grace, J.; Kruijt, B.; Pereira, M. G. P.; Culf, A.; Scott, S. Carbon dioxide transfer over a central Amazonian rain forest. Journal of Geophysical Research. v. 103, n. 31, p. 593-31.612, 1998.

Melillo, J.M.; McGuire, D. A.; Kicklighter, D. W.; Moore, B.; Vorosmarty, C. J.; Schloss, A. L. Global climate change and terrestrial net primary production. Nature. v. 363, p 234-240, 1993.

Shuttleworth, W. J. Evaporation from amazonian rainforest. The Royal Society. v. 233, p. 321-346, 1988.

Tóta, J.; Fitzjarrald, D. R.; Silva-Dias, M. A. F. Exchange of Carbon Between the Atmosphere and the Tropical Amazon Rainforest. The ScientificWorld Journal, v. 2012. p. 305-330, 2012.

Yi, C., (2008), Momentum transfer within canopies. Journal of Applied Meteorology and Climatology, 47, 262-275, doi:10.1175/2007JAMC1667.1.

Yi, C., R. K. Monson, Z. Zhai, D. E. Anderson, B. Lamb, G. Allwine, A. A. Turnipseed, and S. P. Burns (2005), Modeling and measuring the nocturnal drainage flow in a high-elevation, subalpine forest with complex terrain, Journal of Geophysical Research, 110, (D22)303, doi:10.1029/2005JD006282. 